\title{
Reductions in parietal and temporal cerebral metabolic rates for glucose are not specific for Alzheimer's disease
}

\author{
Mark B Schapiro, Pietro Pietrini, Cheryl L Grady, M J Ball, Charles DeCarli, \\ Anand Kumar, Jeffrey A Kaye, James V Haxby
}

\begin{abstract}
Reduction in the regional cerebral metabolic rate for glucose (rCMRglc) in the parietal and temporal regions has been shown in Alzheimer's disease (AD). The specificity of these findings for this disease state is uncertain. We repeatedly measured rCMRglc with positron emission tomography and [18F]2-fluoro-2deoxy-D-glucose in the resting state in a 68 year old man with slowly progressive dementia who, during life, was initially diagnosed as having dementia of the Alzheimer type, then Parkinson disease with dementia, but was found to have only Parkinson's disease at necropsy. Metabolic ratios (rCMRglc/mean grey CMRglc) were significantly $(p<0.05)$ reduced in parietal and temporal regions, as well as in the prefrontal and premotor areas. This pattern was similar in regional distribution and magnitude of the defect to that seen in patients with probable AD. These results suggest that reductions of glucose metabolism in association neocortex in $\mathbf{A D}$ are not specific to the disease process, but may be related to the dementia state.
\end{abstract}

$(\Im$ Neurol Neurosurg Psychiatry 1993;56:859-864)

Laboratory of

Neurosciences, Section

on Brain Aging and

Dementia, National

Institute on Aging,

Clinical Center,

Bethesda, MD 20892,

USA

M B Schapiro

P Pietrini

C L Grady

C DeCarli

A Kumar

J A Kaye

J V Haxby

Dementia Study

Laboratory, Atkinson

Neurobiology of Aging

Unit, Department of

Pathology, University

of Western Ontario,

London M6A 5C1,

Canada

M J Ball

Correspondence to:

Mark B Schapiro, MD

Laboratory of Neurosciences,

National Institute on Aging

Bldg 10 Rm 6C414, 9000

Rockville Pike, Bethesda,

MD 20892, USA.

Received 13 May 1991

and in revised form

1992

Accepted 19 November 1992 ease. He received no medications. There was no family history of AD or PD. Review of systems was notable for impotence. On examination, blood pressure was 140/90 (supine) and 104/76 (standing), and heart rate was 54 (supine) and 64 (standing). There was a mild tremor of outstretched hands.

Laboratory investigations revealed normal serum chemistries, cholesterol, thyroid function test, VDRL, folate, vitamin B12, blood counts, and sedimentation rate. Chest $x$ ray showed borderline cardiomegaly and ECG was normal. EEG showed 7-8 Hz, low amplitude background. CT scan showed mild cortical atrophy. CSF was clear with normal opening pressure.

An extensive battery of neuropsychological tests to assess memory, attention to simple and complex sets, planning, language, visuospatial functions, and overall dementia severity ${ }^{45}$ was administered to the patient five times at roughly annual intervals (table 1 ). To test whether scores differed significantly from that which would be expected in an age, education, and gender-matched control, predicted scores were calculated based on a multiple regression equation derived from 108 healthy controls. These controls ranged in age from 45 to 90 years (mean 63) with 12 to 20 years of education (mean 16.7). Test scores were predicted based on age, gender, and years of education. The difference between actual and predicted score was converted to a $\mathrm{z}$-score by dividing by the standard deviation of control residuals, calculated with a jackknife procedure whereby the predicted score for each control subject was based on a multiple regression equation derived from all controls but that one. To test for significant impairments in $\mathrm{AD}$ patients, the difference between the mean normalised discrepancy and zero was tested with a $t$ test. For the single patient with PD, a score with normalised discrepancy less than $-1.96(p<0.05)$ indicated significant impairment.

On initial evaluation, dementia was mild with significant impairments on two tests of memory, a test of the ability to shift attention between two sequential sets (trails B), planning (Porteus mazes), and visual abstract reasoning (Raven's matrices). Performance on a test of memory for a prose passage (WMS story recall), however, was within normal limits. Whereas all tests of visuospatial construction and immediate visuospatial memory span showed significant impairment at the initial evaluation, all language tests were within normal limits on the first evaluation, and all 
Table 1 Results of longitudinal neuropsychological tests in the healthy controls, Alzheimer's disease patients, and the Parkinson's disease subject. Examinations on fanwary 1987 and Fanuary 1988 coincided with the patient's first and second PET scans, respectively.

\begin{tabular}{|c|c|c|c|c|c|c|c|}
\hline Test & $\begin{array}{l}\text { Controls } \\
(n=11)\end{array}$ & $\begin{array}{l}A D \text { patients } \\
(n=12)\end{array}$ & Nov' '83 & $\begin{array}{l}\text { Parkinson } \\
\text { fan '85 }\end{array}$ & $\begin{array}{l}\text { disease subject } \\
\text { Jan ' } 86\end{array}$ & Jan '87 & Jan' ' 88 \\
\hline \multicolumn{8}{|l|}{ Overall Ability: } \\
\hline $\begin{array}{l}\text { MMSE }(\max =30) \\
\text { WAIS IQ }\end{array}$ & $\begin{array}{l}29 \cdot 8(0 \cdot 4) \\
133(5)\end{array}$ & $\begin{array}{l}23 \cdot 8(2 \cdot 0)^{\star} \\
107(12)^{\star} \\
121(10)^{\star}\end{array}$ & $\begin{array}{l}24 t \\
105 t \\
133 t\end{array}$ & $\begin{array}{r}22 t \\
97 t \\
130 t\end{array}$ & $\begin{array}{r}25 t \\
87 t \\
116 t\end{array}$ & $\begin{array}{r}23 t \\
95 t \\
120 t\end{array}$ & $\begin{array}{r}21 t \\
86 t \\
106 t\end{array}$ \\
\hline \multirow{2}{*}{\multicolumn{8}{|c|}{ Memory: }} \\
\hline \multicolumn{2}{|l|}{ WMS stories } & & & & & & \\
\hline $\begin{array}{l}\text { Immediate recall } \\
\text { Delayed recall }\end{array}$ & $\begin{array}{l}20(7) \\
17(7)\end{array}$ & $\begin{array}{l}9(5)^{\star} \\
4(5)^{\star}\end{array}$ & $\begin{array}{r}12 \\
7\end{array}$ & $\begin{array}{l}5+ \\
4 \dagger\end{array}$ & $\begin{array}{l}7+ \\
2 \dagger\end{array}$ & 14 & 4t \\
\hline \multicolumn{8}{|l|}{ WMS Figures } \\
\hline Immediate recall & $\begin{array}{r}10(3) \\
9(4)\end{array}$ & $\begin{array}{l}3(2)^{\star} \\
1(2)^{\star}\end{array}$ & $1+$ & 1f & $\begin{array}{l}5 \\
0+\end{array}$ & $\begin{array}{l}6 \\
1\end{array}$ & $1+$ \\
\hline \multicolumn{8}{|l|}{ Buschke Test } \\
\hline $\begin{array}{l}\text { Total recall } \\
\text { Delayed recall }\end{array}$ & $\begin{array}{r}64(5) \\
8(1)\end{array}$ & $\begin{array}{l}40(9)^{\star} \\
2(3)^{\star}\end{array}$ & $\begin{aligned} 41+ \\
1+\end{aligned}$ & $\begin{aligned} 41+ \\
3+\end{aligned}$ & $35+$ & $\begin{array}{r}39 f \\
2 f\end{array}$ & $28+$ \\
\hline \multirow{2}{*}{\multicolumn{8}{|c|}{$\begin{array}{l}\text { Complex attention, planning, abstraction: } \\
\text { Trails B (secs) }\end{array}$}} \\
\hline & & & & & & & \\
\hline Stroop interference & $36(8)$ & $14(10)^{\star}$ & 22 & 9+ & $3+$ & $1+$ & $6+$ \\
\hline Porteus mazes (Test, years) & $15 \cdot 8(1 \cdot 1)$ & $12 \cdot 8(4)^{\star}$ & $7 \cdot 5 t$ & $8 \cdot 0+$ & $6.0+$ & 6.0t & $6 \cdot 5 t$ \\
\hline Raven's coloured matrices & $33(3)$ & $25(6)^{\star}$ & $21+$ & $15 t^{\circ}$ & $19+$ & $16 t$ & $13+$ \\
\hline \multicolumn{8}{|l|}{ Language: } \\
\hline WAIS VDQ & $134(9)$ & $117(15)^{\star}$ & 123 & $109 \dagger$ & $108 †$ & $114 \dagger$ & $108 \mathrm{t}$ \\
\hline WAIS MDQ & $124(11)$ & $97(15)^{\star}$ & 109 & 106 & 911 & $97 t$ & 100 \\
\hline Syntax comprehension & $25(1)$ & $19 \cdot 3(3.9)^{\star}$ & 23 & 21 & 19t & $16 t$ & $17 t$ \\
\hline Boston naming & 38 (4) & $31(8)^{\star}$ & 37 & 35 & 35 & 34 & n.a. \\
\hline FAS serial naming & $44(10)$ & $28(14)^{\star}$ & 34 & $11 t$ & $11 t$ & $14 \dagger$ & $16 t$ \\
\hline WRAT oral reading & $16 \cdot 3(2 \cdot 4)$ & $14 \cdot 3(2 \cdot 5)$ & $16 \cdot 5$ & $14 \cdot 4$ & $16 \cdot 8$ & $15 \cdot 0$ & $15 \cdot 3$ \\
\hline \multicolumn{8}{|l|}{ Visuospatial: } \\
\hline WAIIS PDQ & $122(14)$ & $104(17)^{\star}$ & 87† & $73+$ & $60+$ & 70t & $57 t$ \\
\hline Extended range drawing & $20(2)$ & $17(5)^{\star}$ & $10 \dot{x}$ & $12 f$ & $12 \dagger$ & $8 \dot{f}$ & $7 f$ \\
\hline Hiskey-Nebraska blocks & $16(5)$ & $9(4)^{\star}$ & $6 \dot{f}$ & $4 \dagger$ & 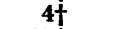 & $3+$ & $3 \dot{t}$ \\
\hline Benton facial recognition & $46(3)$ & $43(6)$ & 43 & $36 t$ & $37 \dagger$ & $36 t$ & $32 f$ \\
\hline Block tapping span & $4.7(0.6)$ & $3.8(0.5)$ & $2 \cdot 8 \dagger$ & $3.0+$ & $2 \cdot 2 t$ & $2 \cdot 5 t$ & $1.5 t$ \\
\hline
\end{tabular}

Values are mean (SD)

AD = Alzheimer's disease; MMSE = mini-mental status examination; WAIS = Wechsler adult intelligence scale; $I Q=$ intelligence quotient; WMS = Wechsler memory scale; VDQ = verbal comprehension deviation quotient; $M D Q=$ memory and freedom from distractibility deviation quotient; WRAT = wide range achievement test; $P D Q=$ perceptual onganisation deviation quotient.

$\star_{\text {Mean in }}^{A D}$ patients less than mean in controls by unpaired $t$ test $(p<0.05)$

†Parkinson subjects's value less than mean in controls by $Z$-score analysis $(p<0.05)$.

but two were normal on the second evaluation. Confrontation naming (Boston naming) and oral reading of irregularly spelled words (WRAT) remained normal on all evaluations. Performance on a test of face discrimination (Benton face recognition) demonstrated significant impairments on the second evaluation and on all evaluations thereafter. Mini-mental state examination (MMSE) score was $24 / 30 .^{6}$ His Hachinski ischaemic score was $1 .^{7}$ The clinical diagnosis at this time was dementia of the Alzheimer type. ${ }^{8}$

At examination on 7 August 1985 there were mild orthostatic blood pressure changes; increased tone in the upper extremities (with mild cogwheeling) which increased with activation $(L>R)$; tremor of outstretched arms; an intermittent, small amplitude fast tongue tremor; diminished rapid finger movements; and mild bradykinesia. His gait was characterised by decreased arm swing, slow pace, moderate flexion posture, and decreased stride length. He was unable to tandem walk. Vibratory sense and two point discrimination were decreased in the toes. Extraocular movements were full. His MMSE score was 22. At this point the diagnosis was thought to be probable Alzheimer's disease with extrapyramidal signs. ${ }^{910}$ A trial of a levodopa/ cardidopa combination (Sinemet) was initiated in September 1986 with improvement of his spontaneous movements. However, this drug was discontinued because of hallucinations, not because of any negative effect on his cognitive performance. Other dopamine agonists were not tried.

At the time of his first PET scan (11
January 1987) his MMSE score was 23. A resting tremor and masked facies were also now present. The clinical diagnosis was changed to Parkinson's disease with dementia, due to the slow progression of cognitive changes (table 1). Very slow deterioration of mental status continued to be noticed at subsequent evaluations, with MMSE scores of 18 on 22 July 1987 and 21 on 11 January 1988 (date of his second PET scan). At his last evaluation on 14 June 1988, his MMSE score decreased to 12 and it was noted that he now was dependent in daily living skills. Annual rates of decline (points/year) on both the WAIS IQ and Mattis dementia rating scale were approximately half the mean rate for patients with $\mathrm{AD}$ and were significant $(p<$ 0.05 ) in comparison with the mean in the healthy controls (WAIS: +0.4 (SD 1.8) for controls, -8.3 (SD 4.4) for $\mathrm{AD}$ patients, -3.8 for the PD patient, respectively. Mattis dementia rating scale: +1.5 (SD 2.1) for controls, -12.4 (SD 6.9) for $\mathrm{AD}$ patients, -6.1 for the PD patients).

Progression of motor abnormalities continued through the course of his illness. Extraocular movements remained full, but saccades were hypometric.

At necropsy by one of us (MJB), the fresh brain weighed 1430 grams. On gross inspection, there was no evidence of cortical atrophy, minimal ventricular dilatation, no atherosclerosis, and no cerebral infarctions. Transverse sections through the brainstem showed striking pallor of the substantia nigra and locus ceruleus. Microscopic examination showed severe depletion of pigmented neu- 
Table 2 Absolute rCMRglc values measured in the healthy volunteers, Alzheimer's disease subjects, and Parkinson's disease subject (first and second PET scans)

\begin{tabular}{|c|c|c|c|c|}
\hline $\begin{array}{l}\text { Brain Region } \\
\text { Absolute Values } \\
\text { (mg/100 g/min) }\end{array}$ & $\begin{array}{l}\text { Controls } \\
(n=19) \\
\text { Mean }(S D)\end{array}$ & $\begin{array}{l}A D \\
(n=12) \\
\text { Mean }(S D)\end{array}$ & $\begin{array}{r}P \\
P E T \text { scan } 1 \\
(01-15-87)\end{array}$ & $\begin{array}{l}P E T \text { scan } 2 \\
(01-21-88)\end{array}$ \\
\hline \multicolumn{5}{|l|}{ Orbito frontal } \\
\hline $\begin{array}{l}\text { right } \\
\text { left }\end{array}$ & $\begin{array}{l}7 \cdot 42(1 \cdot 22) \\
7 \cdot 59(1 \cdot 16)\end{array}$ & $\begin{array}{l}5.84(1.55)^{\star} \\
6.10(1.30)^{\star}\end{array}$ & $\begin{array}{l}5 \cdot 65 \\
6.39\end{array}$ & $\begin{array}{l}5 \cdot 35 \\
5 \cdot 70\end{array}$ \\
\hline \multicolumn{5}{|l|}{ Prefrontal } \\
\hline $\begin{array}{l}\text { night } \\
\text { left }\end{array}$ & $\begin{array}{l}8.76(1.45) \\
8.78(1.50)\end{array}$ & $7.40(0.98)^{\star}$ & $\begin{array}{l}5 \cdot 36+3 \\
4 \cdot 95^{\star} \ddagger\end{array}$ & $\begin{array}{l}5 \cdot 40 \dagger 3 \\
5 \cdot 23^{\star} \ddagger\end{array}$ \\
\hline \multicolumn{5}{|l|}{ Premotor } \\
\hline $\begin{array}{l}\text { right } \\
\text { left }\end{array}$ & $\begin{array}{l}9 \cdot 38(1 \cdot 70) \\
9 \cdot 53(1.56)\end{array}$ & $\begin{array}{l}7 \cdot 48(1 \cdot 18)^{\star} \\
7.58(1.08)^{\star}\end{array}$ & $\begin{array}{l}5 \cdot 21^{\star} \\
5 \cdot 50^{\star}\end{array}$ & $\begin{array}{l}5 \cdot 56 \dagger \\
4 \cdot 80^{\star} \ddagger\end{array}$ \\
\hline \multicolumn{5}{|l|}{ Inferior parietal } \\
\hline left & $8.89(1.74)$ & $6.06(1.38)^{\star}$ & $4 \cdot 58^{\star}$ & $3 \cdot 81^{\star^{7}}$ \\
\hline \multicolumn{5}{|l|}{ Medial parietal } \\
\hline $\begin{array}{l}\text { right } \\
\text { left. }\end{array}$ & $\begin{array}{l}9.53(1.75) \\
9.04(2.02)\end{array}$ & $\begin{array}{l}6.68(0.97)^{\star} \\
6.64(0.82)^{\star}\end{array}$ & $\begin{array}{l}3.69^{\star} \ddagger \\
4 \cdot 34^{\star} \ddagger\end{array}$ & $\begin{array}{l}4 \cdot 65^{\star 3} 3 \\
3 \cdot 63^{\star} \ddagger\end{array}$ \\
\hline \multicolumn{5}{|l|}{ Superior parietal } \\
\hline $\begin{array}{l}\text { right } \\
\text { left }\end{array}$ & $\begin{array}{l}8.48(1.52) \\
8.47(1.56)\end{array}$ & $\begin{array}{l}6.16(1.06)^{\star} \\
6.22(1.04)^{\star}\end{array}$ & $\begin{array}{l}4 \cdot 13^{\star} \\
4 \cdot 19^{\star}\end{array}$ & $\begin{array}{l}4 \cdot 06^{\star} \\
4 \cdot 37^{\star}\end{array}$ \\
\hline \multicolumn{5}{|l|}{ Sensorimotor } \\
\hline $\begin{array}{l}\text { right } \\
\text { left }\end{array}$ & $\begin{array}{l}8.48(1.48) \\
8 \cdot 89(1.62)\end{array}$ & $\begin{array}{l}7.38(1.03) \dagger \\
7.42(0.97)^{\star}\end{array}$ & $\begin{array}{l}5.44 \dagger \\
5.89\end{array}$ & $\begin{array}{l}5 \cdot 19+\ddagger \\
5 \cdot 25 \dagger \S\end{array}$ \\
\hline \multicolumn{5}{|l|}{ Calcarine } \\
\hline $\begin{array}{l}\text { night } \\
\text { left }\end{array}$ & $\begin{array}{l}8.36(1.32) \\
8 \cdot 47(1.31)\end{array}$ & $\begin{array}{l}7.05(1.29)^{\star} \\
7.20(1.24)^{\star}\end{array}$ & $\begin{array}{l}6 \cdot 10 \\
5 \cdot 25^{\star}\end{array}$ & $\begin{array}{l}5 \cdot 38+ \\
4 \cdot 87^{\star}\end{array}$ \\
\hline \multicolumn{5}{|l|}{ Occipital association } \\
\hline right & $\begin{array}{l}8.05(1.42) \\
8.07(1.51)\end{array}$ & $\begin{array}{l}6.95(1 \cdot 00) \dagger \\
6.73(1.13)^{\star}\end{array}$ & $\begin{array}{l}4 \cdot 40^{\star} \ddagger \\
4 \cdot 60 \dagger\end{array}$ & $\begin{array}{l}4 \cdot 54^{\star} \ddagger \\
3 \cdot 81^{\star} \ddagger\end{array}$ \\
\hline \multicolumn{4}{|l|}{ Inferior temporal } & $3 \cdot 80$ \\
\hline \multicolumn{5}{|l|}{ Middle temporal } \\
\hline $\begin{array}{l}\text { right } \\
\text { left }\end{array}$ & $\begin{array}{l}7.99(1.35) \\
8.08(1.28)\end{array}$ & $\begin{array}{l}6.00(0.97)^{\star} \\
6.14(0.86)^{\star}\end{array}$ & $\begin{array}{l}4 \cdot 31^{\star} \\
4 \cdot 92^{\star}\end{array}$ & $\begin{array}{l}4 \cdot 22^{\star} \\
4 \cdot 29^{\star} \S\end{array}$ \\
\hline \multicolumn{5}{|l|}{ Superior temporal } \\
\hline $\begin{array}{l}\text { right } \\
\text { left }\end{array}$ & $\begin{array}{l}8.42(1.52) \\
8.59(1.46)\end{array}$ & $\begin{array}{l}6 \cdot 74(0 \cdot 88)^{\star} \\
6 \cdot 70(1 \cdot 07)^{\star}\end{array}$ & $\begin{array}{l}4 \cdot 42^{\star} \ddagger \\
5 \cdot 08^{\star}\end{array}$ & $\begin{array}{l}4 \cdot 24^{\star} \ddagger \\
4 \cdot 25^{\star} \S\end{array}$ \\
\hline \multicolumn{5}{|l|}{ Anterior cingulate } \\
\hline $\begin{array}{l}\text { right } \\
\text { left }\end{array}$ & $\begin{array}{l}8.66(1.72) \\
8.58(1.44)\end{array}$ & $\begin{array}{l}7.05(0.84)^{\star} \\
7.05(1.10)^{\star}\end{array}$ & $\begin{array}{l}6 \cdot 88 \\
6 \cdot 82\end{array}$ & $\begin{array}{l}6.27 \\
6.97\end{array}$ \\
\hline \multicolumn{5}{|l|}{ Amygdala } \\
\hline $\begin{array}{l}\text { right } \\
\text { left }\end{array}$ & $\begin{array}{l}5.57(0.96) \\
5.66(1.11)\end{array}$ & $\begin{array}{l}4.23(1.35)^{\star} \\
3.76(0.98)^{\star}\end{array}$ & $\begin{array}{l}6 \cdot 65 \\
5 \cdot 77 \S\end{array}$ & $\begin{array}{l}5 \cdot 89 \\
3.97\end{array}$ \\
\hline \multicolumn{5}{|l|}{ Insula } \\
\hline $\begin{array}{l}\text { right } \\
\text { left }\end{array}$ & $\begin{array}{l}8 \cdot 10(1 \cdot 56) \\
8 \cdot 17(1 \cdot 34)\end{array}$ & $\begin{array}{l}6.53(1.71)^{\star} \\
6.62(1.40)^{\star}\end{array}$ & $\begin{array}{l}5 \cdot 35 \\
5 \cdot 35 \dagger\end{array}$ & $\begin{array}{l}5 \cdot 24 \\
4 \cdot 90^{\star}\end{array}$ \\
\hline \multicolumn{5}{|l|}{ Paracentral } \\
\hline $\begin{array}{l}\text { right } \\
\text { left }\end{array}$ & $\begin{array}{l}8 \cdot 26(1 \cdot 77) \\
8 \cdot 36(1 \cdot 67)\end{array}$ & $6.72(0.84)^{\star}$ & $\begin{array}{l}5.92 \\
6.30\end{array}$ & $\begin{array}{l}0.65 \\
5.89\end{array}$ \\
\hline \multicolumn{5}{|l|}{ Hippocampus } \\
\hline $\begin{array}{l}\text { right } \\
\text { left }\end{array}$ & $\begin{array}{l}6 \cdot 75(1 \cdot 15) \\
6 \cdot 78(1.28)\end{array}$ & $\begin{array}{l}5.47(0.81)^{\star} \\
5.20(0.54)^{\star}\end{array}$ & $\begin{array}{l}5 \cdot 10 \\
4 \cdot 41\end{array}$ & $\begin{array}{l}4 \cdot 62 \\
4 \cdot 12 \dagger\end{array}$ \\
\hline Midbrain & $5.37(0.60)$ & $4 \cdot 74(0 \cdot 76) \dagger$ & $4 \cdot 87$ & $5 \cdot 41$ \\
\hline \multicolumn{5}{|l|}{ Caudate nucleus } \\
\hline $\begin{array}{l}\text { right } \\
\text { left }\end{array}$ & $\begin{array}{l}8.82(1.93) \\
9 \cdot 27(1.77)\end{array}$ & $\begin{array}{l}8.11(1.49) \\
8.19(1.67)\end{array}$ & $\begin{array}{l}7 \cdot 85 \\
7 \cdot 08\end{array}$ & $\begin{array}{l}5 \cdot 54 \\
6 \cdot 61\end{array}$ \\
\hline \multicolumn{5}{|l|}{ Lenticular nucleus } \\
\hline $\begin{array}{l}\text { right } \\
\text { left }\end{array}$ & $\begin{array}{l}9 \cdot 54(1.66) \\
9 \cdot 65(1.34)\end{array}$ & $\begin{array}{l}8.43(1.75) \\
8.53(1.55) \dagger\end{array}$ & $\begin{array}{l}8 \cdot 11 \\
8 \cdot 08\end{array}$ & $\begin{array}{l}8 \cdot 43 \\
8 \cdot 24\end{array}$ \\
\hline \multicolumn{5}{|l|}{ Thalamus } \\
\hline left & $9 \cdot 22(1.66)$ & $7.74(1.58) \dagger$ & 8.69 & 6.64 \\
\hline \multicolumn{5}{|l|}{ Cerebellum } \\
\hline $\begin{array}{l}\text { right } \\
\text { left }\end{array}$ & $\begin{array}{l}6.84(1.30) \\
6.88(1.58)\end{array}$ & $\begin{array}{l}6.35(0.57) \\
6.27(0.49)\end{array}$ & $\begin{array}{l}5 \cdot 69 \\
5 \cdot 38\end{array}$ & $\begin{array}{l}4 \cdot 86 \ddagger \\
4 \cdot 37 \ddagger\end{array}$ \\
\hline Global grey matter & $8 \cdot 41(1.37)$ & $6.84(0.82)^{\star}$ & $5 \cdot 47 \dagger$ & $5 \cdot 10^{\star}$ \\
\hline
\end{tabular}

Values of rCMRglc are mean (SD) and are expressed in $\mathrm{mg} / 100 \mathrm{~g}$ tissue/min. Differences between mean values in the Alzheimer's disease and healthy volunteer groups were compared with an unpaired $t$ test.

PD patient was compared to both controls and AD patients by using a $Z$-score analysis.

PD patient was compared to both controls and $\mathrm{AD}$ patier

$\star$ Value differs from mean in controls at $p<0.01$ level.
+ Value differs from mean in controls at $p<0.05$ level.

Value differs from mean in controls at $p<0.05$ level.

$\ddagger$ Value differs from mean in $\mathrm{AD}$ patients at $\mathrm{p}<0.01$ level.
$\S$ Value differs from mean in $\mathrm{AD}$ patients at $\mathrm{p}<0.05$ level.

rons in the substantia nigra, especially in the pars compacta, accompanied by extensive astrocytic gliosis and free neuromelanin pigment. Frequent Lewy bodies were seen in the residual substantia nigra neurons. The remainder of the midbrain showed no significant abnormality. Neurofibrillary tangles were not found. Even more depletion of pigmented nerve cells was shown in the locus ceruleus. The pontine raphe nuclei were normal. In the nucleus basalis of Meynert the average number of nucleolated neurons per $10 \mu \mathrm{M}$ paraffin-embedded section was 38.5 in the anterior division and 13.1 in the intermediate division (the posterior division was not available), a reduction of $90 \%$ and $93 \%$, respectively, in comparison with four non-demented subjects aged 67-72 years. Neither Lewy bodies nor neurofibrillary tangles were found in this sample of nucleus basalis of Meynert. There were no lesions seen in samples of the thalamus with subthalamic nucleus, caudate, and putamen, but there was suggestion of a mild, patchy neuronal loss in the globus pallidus. Within the hippocampal formation, there was a relatively well preserved population of pyramidal neurons, with only a very occasional focus of minimal neuronal dropout. The adjacent amygdala nuclei were also generally within normal limits. The neocortical population of nerve cells, including samples from the middle frontal, inferior temporal, and superior parietal gyri, and calcarine cortex, was well preserved. There were no neurofibrillary tangles or senile plaques in either the neocortex or hippocampal formation.

A monoclonal mouse antibody to ubiquitin (Chemicon International, Inc) showed typical ubiquitin-positive staining in the substantia nigra in this case, while simultaneous staining with normal rabbit serum did not show the positive staining. Lewy bodies or ubiquitinpositive inclusions were not seen in numerouos neocortical regions, including left middle frontal gyrus, left inferior temporal gyrus, and left superior parietal gyrus, hippocampus, corpus striatum, or basal forebrain. Pathology of the spinal cord and sympathetic ganglia was not obtained.

For the PET scan studies, the PD subject was compared with two groups of subjects who were participants in the National Institute on Aging-Laboratory of Neurosciences Studies of Aging and Dementia. ${ }^{111}$ One comparison group included 19 healthy male volunteers (mean age (SD) 64 (6) years, range 57-78), whose MMSE score was $29 \cdot 8$ $(0.4)$. The second group consisted of 12 men with probable Alzheimer's disease $(\mathrm{AD})^{7}$ (mean age (SD): 65 (10) years, range 52-80), whose MMSE score was $23 \cdot 8(2 \cdot 0)$, range 21 to 27 (table 1). MMSE mean scores differed significantly in the two groups ( $p<0.0001)$. These subjects were screened with medical, neurological, and laboratory tests. Subjects had no history of neurological (other than AD in the patient group), psychiatric, or systemic medical disorder. None was taking medication for at least two weeks before the study. All the $\mathrm{AD}$ patients were still alive at the time of present report; therefore, no necropsy data were available to confirm diagnosis of probable AD.

Healthy volunteers and $\mathrm{AD}$ subjects (or their legal guardians) signed an informed consent describing the purpose of the study, the tests performed, and the risks involved. The research was conducted under NIH protocols 81-AG-10 (Parkinson's disease and AD subjects) and 80-AG-26 (controls).

PET scans were performed using procedures previously described in subjects at rest with reduced auditory and visual 
Table 3 The ratio values of rCMRglc to mean calcarine CMRglc measured in the healthy volunteers, Alzheimer's disease subjects, and Parkinson's disease subject (first and second PET scans).

\begin{tabular}{|c|c|c|c|c|}
\hline Brain region & $\begin{array}{l}\text { Controls } \\
(n=19) \\
\text { Mean }(S D)\end{array}$ & $\begin{array}{l}A D \\
(n=12) \\
\operatorname{Mean}(S D)\end{array}$ & $\begin{array}{r}P \\
\text { PET Scan } 1 \\
(01-15-87)\end{array}$ & $\begin{array}{l}\text { ient } \\
\text { PET Scan } 2 \\
(01-21-88)\end{array}$ \\
\hline \multicolumn{5}{|l|}{ Orbitofrontal } \\
\hline right & $0.88(0.08)$ & $0 \cdot 80(0 \cdot 18)$ & $1 \cdot 00$ & 1.04 \\
\hline $\begin{array}{c}\text { left } \\
\text { Prefrontal }\end{array}$ & $0.90(0.05)$ & $0.84(0.15)$ & $1 \cdot 12^{\star}$ & $1 \cdot 11^{\star}$ \\
\hline right & $1.04(0.08)$ & $1.05(0.17)$ & 0.94 & 1.05 \\
\hline left & $1.04(0.08)$ & $1.02(0.14)$ & $0.87 \dagger$ & 1.02 \\
\hline \multicolumn{5}{|l|}{ Premotor } \\
\hline right & $1 \cdot 11(0 \cdot 10)$ & $1.07(0.19)$ & 0.92 & $1 \cdot 08$ \\
\hline left & $1.13(0.09)$ & $1.09(0.21)$ & 0.97 & $0.94 \dagger$ \\
\hline \multicolumn{5}{|l|}{ Inferior parietal } \\
\hline right & $1.03(0.13)$ & $0.92(0 \cdot 14) \dagger$ & $0 \cdot 76 \dagger$ & $0.74 \dagger$ \\
\hline left & $1.05(0.10)$ & $0.86(0.22) \dagger$ & $0.81 \dagger$ & $0 \cdot 74^{\star}$ \\
\hline \multicolumn{5}{|l|}{ Medial parietal } \\
\hline $\begin{array}{l}\text { right } \\
\text { left }\end{array}$ & $1 \cdot 13(0 \cdot 14)$ & $0.95(0.14)^{\star}$ & $0 \cdot 65^{\star}$ & 0.91 \\
\hline $\begin{array}{l}\text { left } \\
\text { Superior parietal }\end{array}$ & $1.07(0 \cdot 13)$ & $0.95(0.15) \dagger$ & $0 \cdot 76 \dagger$ & $0 \cdot 71^{\star}$ \\
\hline \multicolumn{5}{|l|}{ Superior parietal } \\
\hline $\begin{array}{l}\text { right } \\
\text { left }\end{array}$ & $1.01(0.11)$ & $0 \cdot 88(0 \cdot 16)^{\star}$ & $0.73 t$ & $0.79 \dagger$ \\
\hline \multicolumn{5}{|l|}{ Sensorimotor } \\
\hline right & $1.01(0.09)$ & $1.05(0.18)$ & 0.96 & 1.01 \\
\hline left & $1.06(0.10)$ & $1.06(0.21)$ & $1 \cdot 04$ & 1.02 \\
\hline \multicolumn{5}{|l|}{ Occipital association } \\
\hline right & $0.96(0.08)$ & $0.98(0.11)$ & $0.78 \dagger$ & 0.88 \\
\hline $\begin{array}{l}\text { left } \\
\text { Inferior temporal }\end{array}$ & $0.96(0.08)$ & $0.96(0.21)$ & $0 \cdot 81$ & $0 \cdot 74^{\star}$ \\
\hline \multicolumn{5}{|l|}{ Inferior temporal } \\
\hline $\begin{array}{l}\text { right } \\
\text { left }\end{array}$ & $0.76(0.09)$ & $0.72(0.13)$ & - & 0.74 \\
\hline \multicolumn{5}{|l|}{ Middle temporal } \\
\hline right & $0.95(0.08)$ & $0.83(0.09)^{\star}$ & $0.76 \dagger$ & 0.82 \\
\hline left & $0.96(0.07)$ & $0.86(0.14) \dagger$ & 0.87 & 0.84 \\
\hline \multicolumn{5}{|l|}{ Superior temporal } \\
\hline right & $1.00(0.09)$ & $0.97(0.19)$ & $0.78 \dagger$ & 0.83 \\
\hline \multirow{2}{*}{\multicolumn{5}{|c|}{ Anterior cingulate }} \\
\hline & & & & \\
\hline $\begin{array}{l}\text { right } \\
\text { left }\end{array}$ & $\begin{array}{l}1.03(0.09) \\
1.02(0.07)\end{array}$ & $\begin{array}{l}1.01(0.19) \\
1.01(0.22)\end{array}$ & $\begin{array}{l}1.21 \dagger \\
1.20^{\star}\end{array}$ & $\begin{array}{l}1.22 \dagger \\
1.36^{\star}\end{array}$ \\
\hline Posterior cingulate & $1.11(0.11)$ & $1.00(0.14) \dagger$ & $0.70+\S$ & 0.90 \\
\hline \multicolumn{5}{|l|}{ Amygdala } \\
\hline right & $0 \cdot 66(0.08)$ & $0.59(0.15)$ & $1 \cdot 17^{\star} \ddagger$ & $1 \cdot 15^{\star} \ddagger$ \\
\hline left & $0.67(0.08)$ & $0.53(0.13)^{\star}$ & 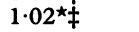 & $0 \cdot 77$ \\
\hline \multicolumn{5}{|l|}{ Insula } \\
\hline right & $0.96(0.10)$ & $0.92(0.24)$ & 0.94 & 1.02 \\
\hline left & $0.97(0.09)$ & $0.94(0.22)$ & 0.94 & 0.96 \\
\hline \multicolumn{5}{|l|}{ Paracentral } \\
\hline right & $0.99(0.11)$ & $0.96(0.19)$ & 1.05 & $1 \cdot 30^{\star}$ \\
\hline left & $0.99(0.09)$ & $0.98(0.15)$ & $1 \cdot 11$ & $1 \cdot 15$ \\
\hline \multicolumn{5}{|l|}{ Hippocampus } \\
\hline right & $0.80(0.08)$ & $0.77(0.09)$ & 0.90 & 0.90 \\
\hline left & $0.80(0.07)$ & $0.74(0.12)$ & $0 \cdot 78$ & $0 \cdot 80$ \\
\hline Midbrain & $0.64(0.07)$ & $0 \cdot 70(0.18)$ & $0 \cdot 86^{\star}$ & $1 \cdot 05^{\star}$ \\
\hline \multicolumn{5}{|l|}{ Caudate nucleus } \\
\hline right & $1.04(0.13)$ & $1 \cdot 15(0 \cdot 23)$ & $1 \cdot 38^{\star}$ & 1.08 \\
\hline left & $1.11(0 \cdot 17)$ & $1 \cdot 16(0.25)$ & $1 \cdot 25$ & $1 \cdot 29$ \\
\hline \multicolumn{5}{|l|}{ Lenticular nucleus } \\
\hline right & $1 \cdot 14(0 \cdot 14)$ & $1 \cdot 19(0 \cdot 17)$ & $1.43 t$ & $1 \cdot 64^{\star} \ddagger$ \\
\hline $\begin{array}{l}\text { lett } \\
\text { Thalamus }\end{array}$ & $1.15(C$ & $1 \cdot 21$ & $1 \cdot 42^{n}$ & $1 \cdot 61 \times 8$ \\
\hline right & $1 \cdot 10(0 \cdot 17)$ & $1 \cdot 14(0 \cdot 16)$ & $1 \cdot 25$ & $1 \cdot 47 \dagger \S$ \\
\hline left & $1 \cdot 10(0 \cdot 17)$ & $1 \cdot 10(0 \cdot 19)$ & $1.53 t$ & $1 \cdot 26$ \\
\hline Cerebellum & & & & \\
\hline right & $0.82(0 \cdot 10)$ & $0.93(0.15)$ & 1.00 & 0.95 \\
\hline left & $0.83(0.11)$ & $0.92(0.15)$ & 0.95 & 0.85 \\
\hline
\end{tabular}

Values are mean (SD).

To compare controls with AD patients an unpaired $t$ test was employed. PD patient was compared with both controls and $\mathrm{AD}$ patients by using a $\mathrm{Z}$-score analysis.

${ }^{\star}$ Value differs from mean in controls at $\mathrm{p}<0.01$ level.

tValue differs from mean in controls at $p<0.05$ level.

$\neq$ Value differs from mean in $A D$ patients at $p<0.01$ level.

$\S$ Value differs from mean in AD patients at $\mathrm{p}<0.05$ level.

inputs $^{12}$ using a Scanditronix PC-1024-7B scanner and [18]2-fluoro-2-deoxy-D-glucose (18FDG). This is a seven slice machine with a transverse resolution (full width at half maximum) of $6 \mathrm{~mm}$ and an axial resolution of 10 $\mathrm{mm}$. Two interleaved transmission scans were performed for correction of attenuation of radiation by the skull. Forty five minutes after intraveneous injection of $5 \mathrm{mCi}$ of $18 \mathrm{FDG}$, two interleaved emission scans were obtained parallel to and from $10-100 \mathrm{~mm}$ above the IOM line. Arterial blood samples were drawn during the uptake period and scan itself to obtain plasma for measurement of radioactivity and glucose. Regional metabolic rates for glucose (rCMRglc) were calculated in units of $\mathrm{mg} / 100 \mathrm{~g} / \mathrm{min}$ using Brooks' modification ${ }^{13}$ of the operational equation of Sokoloff et al. ${ }^{14}$ Regional brain radioactivities were determined using a template of circular regions of interest $8 \mathrm{~mm}$ in diameter.

At time of the first PET scan (15 January 1987) global CMRglc equalled $5 \cdot 47 \mathrm{mg} / 100$ $\mathrm{g} / \mathrm{min}$ in the Parkinson's disease subject compared with 8.41 (SD 1.37) in the healthy volunteer group $(p<0.05)$ and 6.84 (SD 0.82) in the $A D$ group $(p>0.05)$. Using a $Z$ score analysis, ${ }^{15}$ the $P D$ subject showed significant reductions in absolute rCMRglc values compared with healthy volunteers in all cortical regions, except the bilateral orbitofrontal, anterior cingulate, and paracentral regions, right calcarine, and left sensorimotor (table 2). The later scan on 21 January 1988 showed additional deficits in the right calcarine and left sensorimotor regions. There were no significant reductions of glucose metabolism in the thalamus and basal ganglia. A similar pattern of metabolic reduction was seen in the $\mathrm{AD}$ group compared with controls using an unpaired $t$ test, with significant reductions in rCMRglc in all cortical regions. In comparison with the $A D$ group, the $P D$ patient showed significant reductions of absolute rCMRglc values in bilateral prefrontal and medial parietal regions as well as in the right occipital association and right superior temporal areas at the time of the first PET scan. The second scan revealed additional deficits in the left premotor, right inferior parietal, bilateral sensorimotor, left occipital association, left superior temporal regions.

To reduce the intersubject variance associated with absolute values of rCMRglc in healthy controls and to examine patterns of metabolism, ${ }^{1}$ we calculated ratios of rCMRglc to the mean calcarineCMRglc value (table 3 ), a region relatively spared in $\mathrm{AD} .{ }^{16}$ In the $\mathrm{PD}$ subject, relative reductions were shown in the parietal and temporal regions on both scans compared with healthy volunteers. On second PET scan, the left premotor became significantly reduced, whereas the left prefontal region, which was decreased at the time of the first PET scan, was normal. These changes are illustrated in figure 1 , which shows PET images at two levels in the PD subject and a healthy volunteer. Relative reductions in rCMRglc also were shown in parietal and middle temporal regions in $\mathrm{AD}$ subjects compared with healthy volunteers. Both the PD and $\mathrm{AD}$ subjects showed a similar pattern of reduced rCMRglc in the parietal, temporal, and frontal association cortices, whereas there was relative sparing of brain metabolism in primary motor and sensory, and subcortical regions.

\section{Discussion}

This is a case of a slowly progressive dementia in a patient who, during life, was initially diagnosed as having $\mathrm{AD},{ }^{8}$ then $\mathrm{PD}$ with dementia, but was found to have only PD at necropsy. Visual hallucinations, uncommon in early $\mathrm{AD}$, may have suggested the diagnosis of 


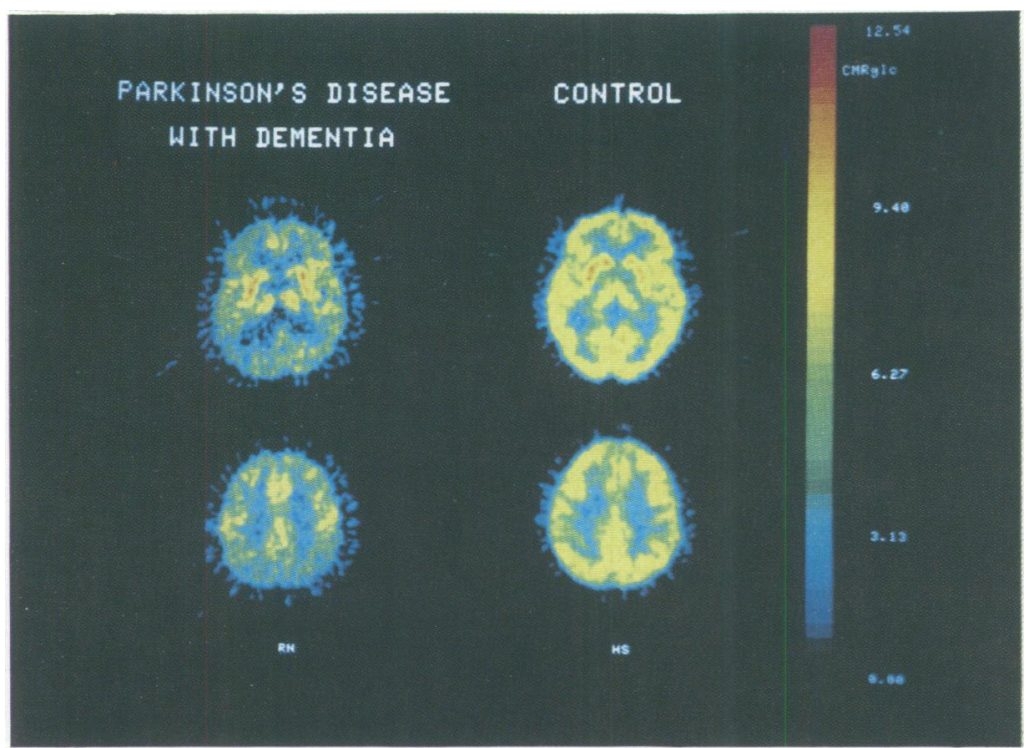

Figure 1 PET images at $45 \mathrm{~mm}$ (top) and $70 \mathrm{~mm}$ (bottom) above the inferior orbital meatal line in a healthy volunteer (right) and the Parkinson's disease subject (left). The colour scale provides values for $\mathrm{rCMRglc}$ in $\mathrm{mg} / 100 \mathrm{~g} / \mathrm{min}$. senile dementia of Lewy body type; necropsy examination, however, ruled out this possibility. In some cases of PD, cognitive impairment may be due to bradyphrenia rather than to a true dementing process. However, neuropsychological testing in our patient showed impairment in multiple cognitive areas, including attention, memory, visuospatial ability, while longitudinal studies showed a progressive cognitive decline, similar to that seen in $\mathrm{AD}$ patients. In comparison with previous reports of PET scanning with $18 F D G$ in early $A D$, and to a mild $A D$ group with comparable MMSE scores in this study, this subject showed similar regional distribution and magnitude of defect in glucose metabolism in the parietal and temporal cortical association areas, with relative sparing of primary neocortex and subcortical regions. Further, these results were replicated on PET scans one year apart. Additional relative reductions in glucose metabolism in prefrontal and premotor regions were found in this subject. Although not among the metabolic findings usually seen in the early $A D$, such reductions have been described in later stages of $\mathrm{AD}^{4}$ These findings suggest that reductions of glucose metabolism in parieto/temporal and frontal association neocortex in $\mathrm{AD}$ are not specific to the disease process, but maybe related to the dementia state.

Such a metabolic pattern has also been described in diseases other than $\mathrm{AD}$, but neuropathology was not always available. In one patient with PD with moderate dementia, who was scanned while on medication, relative reductions of parietal/temporal metabolism were noted; specific reductions of parietal/temporal metabolism were not found in three other cases of PD and dementia in the same report. ${ }^{17}$ In addition, whole brain and bilateral parietal/temporal reductions in metabolism have been described in a patient with normal pressure hydrocephalus whose dementia improved after CSF shunt surgery. ${ }^{18}$ Unlike these two previous examples of reductions of parietal/temporal metabolism in diseases other than $\mathrm{AD}$, our case was confirmed not to have $\mathrm{AD}$ by autopsy. In addition, asymmetric reductions of temporal metabolism have been reported in a necropsy confirmed case of Creutzfeldt-Jakob disease. However, parietal metabolism was not examined. ${ }^{19}$ Patients with vascular dementia (VD) also showed patterns of reduced parietal/temporal and frontal metabolism similar to those seen in $\mathrm{AD}$ patients. ${ }^{20}$ Unlike $\mathrm{AD}$ patients, however, patients with VD had significantly reduced metabolic values also in subcortical structures, such as the basal ganglia, thalami, and cerebellum. Moreover, none of the VD patients had necropsy to confirm diagnosis and exclude a coexistent $\mathrm{AD}$ process.

Unlike the $\mathrm{AD}$ patients, the $\mathrm{PD}$ patient showed normal glucose metabolism and absence of neuropathology in the amygdala nuclei. This may explain, in part, the finding that two short memory tests were normal at the time of the first PET exam.

The cause of the dementia in PD remains uncertain. The numbers of senile plaques and neurofibrillary tangles (NFTs) usually do not differ between PD patients with and without dementia and appropriate controls. ${ }^{21-23}$ An occasional case of PD and dementia has been described with concomitance of both $\mathrm{AD}$ and PD at necropsy, but these are thought to be the exception. In our case no such pathological changes of $\mathrm{AD}$ were present. Further, no cortical Lewy bodies were noted, as described by Clark ${ }^{24}$ in patients with progressive dementia clinically thought to be $\mathrm{AD}$. Rather, our case showed neuropathological changes characteristic of idiopathic PD.

Previous work has shown that resting regional hypometabolism with 18FDG PET in life correlates with regional NFT density postmortem in five of six necropsy proved cases of $\mathrm{AD},{ }^{25}$ suggesting that the hypometabolism in $\mathrm{AD}$ results from dysfunction of neurons in regions of high NFT density. Our case suggests that regional cerebral hypometabolism also may result from neuronal dysfunction which is not related to the formation or accumulation of NFTs. Primary damage to several subcortical systems with cortical projections may contribute to cortical hypometabolism. Cortical cholinergic abnormalities usually occur in Parkinson's disease with dementia in the absence of $\mathrm{AD}$ pathology. ${ }^{23}$ This has been attributed to primary loss of neurons in the subcortical Meynert nucleus, ${ }^{22}$ also seen in our case, which give rise to cortical cholinergic fibres. However, experimental unilateral and bilateral lesions in the nucleus basalis in rats ${ }^{26}$ and in non-human primates ${ }^{27}$ only have caused temporary disturbances in brain metabolism.

As outlined by Rapoport, ${ }^{28} \mathrm{PD}$ is a phylogenic disease of system II, one of several groups of functionally and anatomically related brain regions that underwent dispro- 
portionate expansion during recent evolution of primates, particularly humans. The regions associated in system II, including substantia nigra, basal ganglia, thalamus, and frontal association and motor neocortical areas, participate in several functionally segregated circuits which subserve cognition as well as movement. ${ }^{29}$ The basal ganglia are linked with association cortex, by several of these segregated circuits. A major subcortical component of system II that is disrupted in PD is the nigrostriatal system. Dementia is inversely related to the neuronal cell count in the medial part of the substantia nigra. ${ }^{30}$ The cells in this portion generally project to the caudate, and along with cells in the ventral tegmental area which also are lost in PD, project to cortical and limbic regions. Therefore, significant involvement of parts of the segrated circuitry of system II may result in dementia and cortical hypometabolism. The interaction between altered dopamine and cholinergic function may also have together resulted in the dementia and metabolic abnormalities in our subject.

Dementia and an extrapyramidal syndrome may be seen in, among other diseases, $\mathrm{AD},{ }^{9}$ $P D$ with $A D$, and, as in this case, $P D$ without AD. PET with $18 F D G$ has shown similar reductions in parietal/temporal metabolism in cases from each of these disease categories and therefore has not allowed differentiation of one entity from another. Preliminary work has suggested that PET with [18F]6-fluoro-Ldopa (6-FD) differentiates clinically diagnosed Alzheimer's disease with extrapyramidal features from Parkinson's disease with dementia. ${ }^{31}$ However, it is not known if 6-FD will allow differentiation of demented Parkinson's disease with and without the neuropathological changes of Alzheimer's disease.

1 Haxby JV, Grady CL, Duara R, Schlageter N, Berg G, Rapoport SI. Neocortical metabolic abnormalities precede nonmemory cognitive defects in early Alzheimer'stype dementia. Arch Neurol 1986;43:882-5.

2 Grady CL, Haxby JV, Schlageter NL, Berg G, Rapoport SI. Stability of metabolic and neuropsychological asymmetries in dementia of the Alzheimer type. Neurology 1986;36:1390-2.

3 Schapiro MB, Grady CL, Ball MJ, DeCarli C, Rapoport SI. Reductions in parieto/temporal cerebral glucose SI. Reductions in parieto/temporal cerebral glucose
metabolism are not specific for Alzheimer's disease. metabolism are not specific fo

4 Haxby JV, Grady CL, Koss E, et al. Heterogeneous anterior-posterior metabolic patterns in dementia of the Alzheimer type. Neurology 1988;38:1853-63.

5 Haxby JV, Grady CL, Koss E, et al. Longitudinal study of cerebral metabolic asymmetries and associated neuropsychological patterns in early dementia of the Alzheimer type. Arch Neurol 1990;47:753-60.

6 Folstein MF, Folstein SI, McHugh PR. 'Mini Mental State'-a practical method for grading the cognitive state of patients for the clinician. $\mathcal{F}$ Psychiatr Res 1976;12: 189-98.

7 Hachinski VC, Iliff LD, Zilhka E, et al. Cerebral blood flow in dementia. Arch Neurol 1975;32:632-7

8 American Psychiatric Association Task Force on Nomenclature and Statistics. Diagnostic and statistical manual for mental disorders. 3rd ed. Washington, DC: American Psychiatric Association, 1980.

9 Kaye JA, May C, Daly E, et al. Cerebrospinal fluid monoamine markers are decreased in dementia of the Alzheimer type with extrapyramidal features. Neurology Alzheimer type

10 McKhann G, Drachman D, Folstein M, Katzman R, Price $\mathrm{D}$, Stadlan EM. Clinical diagnosis of Alzheimer's disease: report of the NINCDS-ADRDA work group under the auspices of Department of Health and Human Services task force on Alzheimer's disease. Neurology 1984;34:939-44

11 Duara R, Margolin RA, Robertson-Tchabo EA, et al. Cerebral glucose utilization as measured with positron emission tomography in 21 resting healthy men between the ages of 21 and 83 years. Brain 1983;106:761-75.

12 Swedo SE, Schapiro MB, Grady CL, et al. Cerebral glucose metabolism in childhood-onset obsessive-compulsive disorder. Arch Gen Psychiatry 1989;46:518-23.

13 Brooks RA. Alternative formula for glucose utilization using labeled deoxyglucose. F Nucl Med 1982;23:538-9.

14 Sokoloff L, Reivich M, Kennedy C, et al. The (14C)deoxyglucose method for the measurement of local cerebral glucose utilization: theory, procedure and normal values in the conscious and anesthetized albino rat. $f$ values in the conscious and
Neuroch 1977;28:897-916.

15 Colton T. Statistics in medicine 1st ed. Boston: Little, Brown and Company, 1974:84-7.

6 Kumar A, Schapiro MB, Grady CL, et al. High-resolution PET studies in Alzheimer's disease. Neuropsychopharmacology 1991;4:35-46.

17 Kuhl DE, Metter EJ, Riege WH. Patterns of local cerebral glucose utilization determined in Parkinson's disease by the $[18 \mathrm{~F}]$ fluorodeoxyglucose method. Ann Neurol 1984;15:419-24.

18 Friedland RP. 'Normal'-pressure hydrocephalus and the saga of the treatable dementias. $\mathcal{F A M A} 1989 ; 18$ : saga of

19 Friedland RP, Prusiner SB, Jaqust WJ, Budinger TF, Davis RL. Bitemporal hypometabolism in CreutzfeldtJakob disease measured by positron emission tomography with $[18 \mathrm{~F}]-2$-fluorodeoxyglucose. F Comput Assist Tomogr 1984;8:978-81.

20 Mielke R, Herholz K, Grond M, Kessler J, Heiss W-D. Severity of vascular dementia is related to volume of metabolically impaired tissue. Arch Neurol 1992; 49:909-13.

21 Ball MJ. The morphological basis of dementia in Parkinson's disease. Can F Neurol Sci 1984;11:180-4.

22 Mann DMA, Yates PO. Pathological basis for neurotransmitter changes in Parkinson's disease. Neuropathol Appl Neurobiol 1983;9:3-19.

23 Perry RH, Tomlinson BE, Candy JM, et al. Cortical cholinergic deficit in mentally impaired Parkinsonian pholinergic defic

24 Clark AW, White CL, Manz HJ, et al. Primary degenerative dementia without Alzheimer pathology. Can $f$ Neurol Sci 1986;13:462-70.

25 DeCarli C, Atack JR, Ball MJ, et al. Post-mortem regional neurofibrillary tangle densities but not senile plaque densities are related to regional cerebral metabolic rates for glucose during life in Alzheimer's disease patients. Neurodegeneration 1992;1:11-9.

26 Orzi F, Diana G, Casamenti F, Palombo E, Fieschi C. Local cerebral glucose utilization following unilateral and bilateral lesions of the nucleus basalis magnocellularis in the rat. Brain Res 1988;462:99-103.

27 Kiyosawa M, Baron JC, Hamel E, et al. Time course of effects of unilateral lesions of the nucleus basalis of meynert on glucose utilization by the cerebral cortex. meynert on glucose utilizat

28 Rapoport SI. Integrated phylogeny of the primate brain, with special reference to humans and their diseases. Brain Research Reviews 1990;15:267-94.

29 Alexander GE, DeLong MR, Strick PL. Parallel organization of functionally segregated circuits linking basal ganglia and cortex. Annu Rev Neurosci 1986;9:357-81.

30 Rinne JO, Rummukainen J, Paljarvi L, Rinne U. Dementia in Parkinson's disease is related to neuronal loss in the medial substantia nigra. Ann Neurol 1989;26:47-50.

31 Tyrrell PJ, Sawle GV, Frackowiak RSJ, Rossor MN. Extrapyramidal signs in dementia of the Alzheimer-type: 1989;9:S568. 